\title{
UPPER BOUNDS ON THE DIMENSION OF EXTENDIBILITY OF SUBMANIFOLDS IN $C^{n}$
}

\section{STEPHEN J. GREENFIELD}

1. Introduction. Suppose $K$ is a subset of $C^{n}$. Then $\mathfrak{F C}(K)$ is the collection of all functions holomorphic in a neighborhood of $K$. We say that $K$ is extendible to a connected set $K^{\prime}$ of $C^{n}$ if $K_{\neq}^{c} K^{\prime}$, and the natural restriction map from $\mathcal{H C}\left(K^{\prime}\right)$ to $\mathcal{H}(K)$ is onto.

In the special case that $K$ is a submanifold, $M$, of $C^{n}$ it is interesting to ask for "geometric" conditions on $M$ that insure results on extendibility. Such results can be proven for $C$ - $R$ submanifolds of $C^{n}$. These manifolds also have interesting applications to partial differential equations. (See [1], [2], for details of what follows.)

$T\left(C^{n}\right) \otimes C$ has a splitting into two equal-dimensional subbundles, $H\left(C^{n}\right)$ and $A\left(C^{n}\right)$, obtained from the complex structure of $C^{n}$. $H\left(C^{n}\right)_{p}$ is generated by $\partial /\left.\partial z_{j}\right|_{p}, 1 \leqq j \leqq n$, and is called the holomorphic tangent bundle of $C^{n}$. $A\left(C^{n}\right)$, the antiholomorphic tangent bundle, is the conjugate of $H\left(C^{n}\right)$ in $T\left(C^{n}\right) \otimes C$.

If $M$ is a differentiable submanifold of $C^{n}, M$ is called a $C-R$ submanifold of $C^{n}$ if $H(M)=T(M) \otimes C \cap H\left(C^{n}\right)$ is a vector bundle. If $M$ is a $C$ - $R$ manifold, then $A(M)=T(M) \otimes C \cap A\left(C^{n}\right)$ is also a vector bundle. $H(M) \cap A(M)=0$, and $H(M)$ (resp. $A(M)$ ) is involutive. The Levi algebra of $M, \mathfrak{L}(M)$, is the Lie subalgebra of complex vector fields generated by sections of $A(M)$ and $H(M)$. We make the assumption that the dimension of $\mathfrak{L}(M)$ is constant. Then $\mathscr{L}(M)$ is the algebra of sections of a vector bundle $V$, and $V \supset H(M)+A(M)$. Let $e=$ fiber $\operatorname{dim}_{C} V /(H(M)+A(M))$. $e$ is called the excess dimension of $\mathfrak{L}(M)$, ex $\operatorname{dim} \mathfrak{L}(M)$.

Now max $(\operatorname{dim} M-n, 0) \leqq$ fiber $\operatorname{dim}_{C} H(M) \leqq n$. If fiber $\operatorname{dim}_{C} H(M)$ $=\max (\operatorname{dim} M-n, 0), M$ is called generic. There are two results:

Theorem (Nirenberg ANd Wells [2]). If $M$ is a compact generic $C$ - $R$ submanifold of $C^{n}$, and $\operatorname{dim} M \leqq n$, then $M$ is not extendible.

Theorem ([1]). If $M$ is a generic $C$ - $R$ submanifold of $C^{n}$, and $\operatorname{dim} M>n$, then $M$ is locally extendible to a set containing a differentiable manifold $N$, with $\operatorname{dim} N=\operatorname{dim} M+e$. If $e=0$, then $M$ is locally holomorphically convex.

Received by the editors May 23, 1968. 
(We say a set is locally extendible if each sufficiently small open subset of it is extendible. A set $K$ is locally holomorphically convex if, for any $p \in K, K \cap B$ is not extendible, for $B$ a sufficiently small open ball in $C^{n}$ centered at $p$.)

In the following section we prove a stronger version of the second theorem above for real analytic $C$ - $R$ submanifolds of $C^{n}$, by removing the restriction "generic" and establishing an upper bound on the dimension of local extendibility. We comment on the possibility of proving the theorem for differentiable $C$ - $R$ submanifolds.

Conversations with Professor Hugo Rossi during the preparation of this paper proved invaluable.

2. Real analytic $C-R$ submanifolds. If $M$ is a $C-R$ submanifold of $C^{n}$, then the $C-R$ codimension of $M, C-R \operatorname{codim} M$, is $\operatorname{dim} M$-fiber $\operatorname{dim} H(M)$. If $M$ is generic and $\operatorname{dim} M \geqq n$, then $\operatorname{codim} M$ in $C^{n}=C-R$ $\operatorname{codim} M$.

If $(M, H(M))$ and $\left(M^{\prime}, H\left(M^{\prime}\right)\right)$ are $C$ - $R$ manifolds, a $C$ - $R$ map $f: M \rightarrow M^{\prime}$ is a differentiable map so that $d f(H(M)) \subset H\left(M^{\prime}\right)$. If $M$ is a $C$-R submanifold of $C^{n}$, the restriction of any element of $\mathscr{F C}(M)$ to $M$ is a $C-R$ map from $M$ to $C$.

THEOREM. Let $M$ be a nontrivial real analytic $C$ - $R$ submanifold of $C^{n}$ (so $\left.H(M) \neq 0\right)$. If $e=0, M$ is locally holomorphically convex. If $e>0, M$ is locally extendible to a set containing a manifold $N$, with $\operatorname{dim} N=\operatorname{dim} M+e . M$ is not locally extendible to a set of dimension greater than $\operatorname{dim} M+e$.

Proof. Suppose $M$ is a real analytic $C$ - $R$ submanifold of $C^{n}$, and $\operatorname{dim} M=k$, and $C-R \operatorname{codim} M=l$. Then, if we select $p \in M$, we can find $m\left(=\frac{1}{2}(k+l)\right)$ linear combinations $S_{1}, \ldots, S_{m}$ of the coordinate functions $z_{1}, \ldots, z_{n}$ so that the map $S: C^{n} \rightarrow C^{m}$ given by $S$ $=\left(S_{1}, \ldots, S_{m}\right)$ imbeds $M$ near $p$ as a generic $C-R$ submanifold of $C^{m}$. We restrict our attention to that part of $M$ which is imbedded generically. Then (using a complexification argument due to Tomassini [3]) any real analytic $C-R$ map $f: M \rightarrow C$ is the restriction of a holomorphic function defined in a neighborhood of $S(M)$ in $C^{m}$.

If we suppose ex $\operatorname{dim} \mathfrak{L}(M)=e>0$, then ex $\operatorname{dim} \mathfrak{L}(S(M))=e$. We must show that $M$ is extendible to a set $L$ containing an $(e+k)$ dimensional manifold. If $f \in \mathcal{H}(M)$, then $\left.f\right|_{M}: M \rightarrow C$ is a $C$ - $R$ map. So there is $f^{*} \in \mathcal{H C}(S(M))$ with $\left.f^{*}\right|_{S(M)}=\left.\left.f\right|_{M} \circ S^{-1}\right|_{S(M)} . f^{*}$ extends to a set $L^{*}$ (since $S(M)$ is generic) and $L^{*}$ contains an $(e+k)$ dimensional manifold. Consider now the functions $z_{1}, \ldots, z_{n} \in \mathcal{H C}(M)$. There are associated $z_{1}^{*}, \ldots, z_{n}^{*} \in \mathcal{F}(S(M))$ which extend to $L^{*}$. We define $L: P$ 
$=\left(p_{1}, \ldots, p_{n}\right) \in L$ when there is $q \in L^{*}$ with $p_{j}=z_{j}^{*}(q), 1 \leqq j \leqq n$. By the way we constructed the map $S$, we see that $L$ must contain an $(e+k)$ dimensional manifold since $L^{*}$ does. We define an extension of $f$ to $L$ by taking the extension of $f^{*}$ and transporting back to $L$. By the way $L$ was constructed this is insured to be an analytic function of $z_{1}, \ldots, z_{n}$.

If $e=0$, a similar argument will show that $M$ is locally holomorphically convex. Or, there is also a simple complexification argument for this case.

To complete the remaining assertions of the theorem, we show that there is a real analytic $C-R$ manifold, $N$, with $\operatorname{dim} N=(k+e)$, and ex $\operatorname{dim} \mathfrak{L}(N)=0$, so that

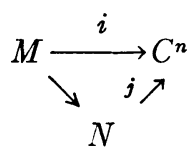

(a diagram of $C$ - $R$ maps) commutes, and each map is of maximal rank ( $i$ is the natural imbedding). The germ of $N$ at $M$ is called the germ of the minimal flattening of $M$ in $C^{n}$, and is unique.

How to obtain $N$ : consider the 'abstract' complexification of $M$, $M_{C} . M_{C}$ is a complex manifold with $M$ a totally real, real analytic submanifold of $M_{C}$, and $\operatorname{dim}_{C} M_{C}=\operatorname{dim} M$. ("Totally real" means "having no holomorphic tangent vectors.") $T(M)_{p} \otimes C=T\left(M_{C}\right)_{p}$. We extend $H(M)$ and $A(M)$ to vector subbundles of $T\left(M_{C}\right)$, perhaps shrinking $M_{C}$ as a neighborhood of $M$. Call these bundles $H^{\prime}$ and $A^{\prime}$. Then $A^{\prime}$ is the conjugate of $H^{\prime}$, and $H^{\prime}$ (resp. $A^{\prime}$ ) is involutive. Let $\mathscr{L}^{\prime}$ be the Lie algebra generated by sections of $H^{\prime}$ and $A^{\prime}$. Then $\mathscr{L}^{\prime}$ is a distribution of constant fiber dimension, since $\mathscr{L}(M)$ is. Let $N$ be the union of all maximal integral submanifolds of $\mathscr{L}^{\prime}$ which intersect $M . N$ is the desired 'locally flat' manifold. The map $j$ is induced by $z_{1}, \ldots, z_{n}$ on $M$ extended to $M_{C}$ and restricted to $N$.

(By a similar method we could also construct the minimal complexification of $M$ in $C^{n}$-the smallest germ of a complex submanifold of $C^{n}$ containing $M$. Something like this is also done in Tomassini [3].)

So $M$ is a subset of a locally holomorphically convex set $N$ (since ex $\operatorname{dim} \mathfrak{L}(N)=0$ ), with $\operatorname{dim} N=\operatorname{dim} M+e$. Therefore $M$ is not locally extendible to a set of dimension greater than $\operatorname{dim} M+e$.

We can also prove a nongeneric extendibility theorem for differentiable $C-R$ submanifolds with $C-R \operatorname{codim}=1$, using a result of Nirenberg and Wells. (Let $Q(K)$ be the uniformly closed algebra of functions on $K$ generated by restrictions to $K$ of functions in $\mathfrak{F C}(K)$.) 
TheOREm [2]. If $M$ is a differentiable hypersurface of $C^{n}$, and $p \in M$, then any sufficiently small compact neighborhood $K$ of $p$ in $M$ has the following property: the uniformly closed algebra of functions generated by restriction to $K$ of $C-R$ fucctions on $M$ is identical with $a(K)$.

We also need:

Lemma. Suppose a compact set $K$ is extendible to a compact set $K^{\prime}$. Then every element of $Q(K)$ is the restriction of a unique function in $a\left(K^{\prime}\right)$.

Then we can get:

THEOREM. Let $M$ be a nontrivial $C-R$ submanifold of $C^{n}$, with $C-R$ $\operatorname{codim}=1$. If $e=\operatorname{ex} \operatorname{dim} \mathfrak{L}(M)$ is 0 , then $M$ is locally holomorphically convex. If $e=1, M$ is locally extendible to a set containing a submanifold $N$, and $\operatorname{dim} N=\operatorname{dim} M+1 . M$ is not locally extendible to a set of dimension greater than $\operatorname{dim} M+1$. (Of course, $e$ is 0 or 1 .)

Proof. Suppose $\operatorname{dim} M=k$. Then, as before, we can find $m=\frac{1}{2}(k+1)$ complex-valued $C-R$ functions $S_{1}, \ldots, S_{m}$ so that $S=\left(S_{1}, \ldots, S_{m}\right)$ is a $C-R$ imbedding of $M$ as a hypersurface of $C^{m}$ near some point $p \in M$. If $e>0$, then $S(M)$ is extendible to a set $L^{*}$ containing a $(k+1)$ dimensional manifold. To transport $L^{*}$ back to $C^{n}$, proceed as in the real analytic case, but use the preceding theorem and lemma instead of the complexification argument.

The function $\left.z_{j}\right|_{M}(1 \leqq j \leqq n)$ is a $C-R$ function; by restricting to a suitable compact neighborhood of $p$, we can find extensions $z_{j}^{*}$ of $z_{j}$ (considered on $S(M)$ ) to some compact subset of $L^{*}$ containing a $C^{m}$-open set. These values of $z_{j}^{*}$ furnish the desired subset $L$ of $C^{n}$, and the extension of any function to $L$ is obtained in the obvious way.

Since we see that functions in $\mathcal{F}(M)$ are locally approximable on $M$ (in small enough compact sets) by analytic functions in $C^{m}, M$ is not locally extendible to a set of dimension greater than $m$.

If $e=0$, then the theorem is given in [1].

Some points worthy of further investigation should be noted. Can the theorem of Nirenberg and Wells (closure of $C$ - $R$ functions $=a(K)$ ) be generalized to higher codimensional submanifolds? Then we could prove theorems similar to the above in higher codimension. If a submanifold is locally holomorphically convex (resp. extendible to a set of dimension at most $k$ ) is it holomorphically convex (resp. extendible to a set of dimension at most $k$ )? This is a higher codimensional analogue of the Levi problem, and seems to be difficult. 


\section{REFERENCES}

1. S. Greenfield, Cauchy-Riemann equations in several variables, Ann. Scuola. Norm. Sup. Pisa 22 (1968), 275-314.

2. R. Nirenberg and R. O. Wells, Approximation theorems on differentiable submanifolds of a complex manifold, Trans. Amer. Math. Soc. 142 (1969), 15-35.

3. G. Tomassini, Tracce delle funzioni olomorfe sulle sottovarietd analitiche reali d'una varietà complessa, Ann. Scuola Norm. Sup. Pisa 20 (1966), 31-44.

Massachusetts Institute of Technology 